Publ. RIMS, Kyoto Univ.

20 (1984), 839-852

\title{
On the Stable Hurewicz Image of Some Stunted Projective Spaces, I
}

\author{
Dedicated to Professor N. Shimada on his 60th birthiay
}

By

Mitsunori IMAOKA* and Kaoru MoRISUGI*

\section{$\S 1$. Introduction}

Let $Z$ be the set of integers, and $Z\left\{a_{1}, a_{2}, \cdots\right\}$ denote a free abelian group with basis $\left\{a_{1}, a_{2}, \cdots\right\}$. If $h: G \rightarrow H$ is a homomorphism between groups, then we denote its image, its kernel and its cokernel by $\operatorname{Im} h$ Ker $h$ and Coker $h$ respectively.

Let $H P^{n}$ (resp. $\left.C P^{n}\right)(0 \leqq n \leqq \infty)$ be the quaternionic (resp. complex) n-dimensional projective space, and $H P_{k}^{n}=H P^{n} / H P^{k-1}$ (resp. $\left.C P_{k}^{n}=C P^{n}, C P^{k-1}\right)(1 \leqq k \leqq n)$ be the stunted projective space. Then, as is well known,

$$
\tilde{H}_{*}\left(H P_{k}^{n} ; Z\right)=Z\left\{\beta_{k}, \beta_{k+1}, \cdots, \beta_{n}\right\},
$$

where $\beta_{\imath} \in H_{11}\left(H P_{k}^{n} ; Z\right)(k \leqq i \leqq n)$ are the standard generators.

Let

$$
h_{n, k}: \pi_{1 n}^{s}\left(H P_{k}^{\infty}\right) \longrightarrow H_{4 n}\left(H P_{k}^{\infty} ; Z\right)
$$

be the stable Hurewicz homomorphism. Then we denote the order of Coker $h_{n, k}$ by $\left|h_{n, k}\right|$. Thus, $\operatorname{Im} h_{n, k}$ is the subgroup generated by $\mid h_{n} k^{\prime} \xi_{1}$. equivalently. $\left|h_{n, k}\right|$ is equal to the stable order of the attaching map of the top cell of $H P_{h}^{n}$.

D. M. Segal [10] has shown that

$$
\left|h_{n, 1}\right|=(2 n) ! / a(n) \quad(n \geqq 1),
$$

where $a(n)=1$ if $n$ is even and $a(n)=2$ if $n$ is odd. In this paper we investigate the order $\left|h_{n, 2}\right|$.

Let $\nu_{2}(i)$ be the exponent of 2 in the prime power decomposition of an integer $i$. Then our main result is stated as follows:

Theorem A. Let $n \geqq 2$. Then

$$
\nu_{2}\left(\left|h_{n, 2}\right|\right)=\nu_{2}(a(n)((2 n) !) / 8),
$$

Communicated by N. Shimada, June 24, 1983.

* Department of Mathematics, Faculty of Education, Wakayama Lniversity, Wakayama 640, Japan 
where $a(n)=1$ if $n$ is even and $a(n)=2$ if $n$ is odd.

Let $p: H P_{2}^{r} \rightarrow H P_{n}^{n}=S^{4 n}$ be the collapsing map, and $p_{*}: \pi_{4 n}^{s}\left(H P_{\underline{2}}^{n}\right) \rightarrow \pi_{1 n}^{s}\left(S^{1 n}\right)$ be the induced homomorphism. Then by definition, $\left|h_{n, 2}\right|$ is equal to the order of Coker $p_{*}$.

Analogously, let $q: C P_{n-k}^{n-1} \rightarrow C P_{n-1}^{n-1}=S^{2 n-2}(1 \leqq k<n)$ be the collapsing map, and $q_{*}: \pi_{2 n-2}^{s}\left(C P_{n-k}^{n-1}\right) \longrightarrow \pi_{2 n-2}^{s}\left(S^{2 n-2}\right)$ be the induced homomorphism. Then, we denote the order of Coker $q_{*}$ by $U(n, k)$. For some $(n, k)$, these numbers $U(n, k)$ are investigated by various authors (cf. [3], [8], [9], [4], [13]).

As an application of Theorem $\mathrm{A}$ and its proof, we have the following:

Theorem B. Let $n \geqq 2$. Then

$$
\nu_{2}(U(2 n+1,2 n-2))=\nu_{2}((2 n) ! / 4) .
$$

Remark. According to Knapp $[4 ;(7.45)]$, the odd primary components of $\left|h_{n, 2}\right|$ and $U(2 n \div-1,2 n-2)$ are already known. $U(2 n, 2 n-3)$ is also determined by Walker [13].

In our forthcoming paper we shall investigate the analogous problems for the quaternionic quasi-projective space, and apply to the complex projective space.

Throughout this paper we use the following notations:

(1.2) For a pointed space $X, \Sigma^{n} X$ denotes the $n$ fold iterated reduced suspension of $X$. As we shall work only in the stable category, for a space $X$, its suspension spectrum is also denoted by the same letter $X$, and, for spaces $X$ and $Y$, a map $h: X \rightarrow Y$ denotes the degree 0 map between their suspension spectra. Moreover, we denote the stable homotopy class $[h] \in\{X, Y\}$ of a map $h$ simply by the same letter $h$.

(1.3) In the stable stems $\pi_{*}^{s}\left(S^{0}\right)$, we denote a generator of $\operatorname{Im} J_{1^{k-1}}$ by $j_{4 k-1}$. where $J_{l}$ is the stable $J$-homomorphism $\pi_{l}(S O) \rightarrow \pi_{l}^{s}\left(S^{0}\right) \quad(l=4 k-1)$. For classes $\alpha, \beta$ and $\gamma$ in $\pi_{*}^{s}\left(S^{0}\right)$ satisfying $\alpha \beta=\beta \gamma=0$, we denote the Toda bracket [12] of them by $\langle\alpha, \beta, \gamma\rangle$. We refer to [12] for various properties on the stable homotopy groups of spheres.

(1.4) We denote the Adams $e_{R}^{\prime}$-invariant [1] of a class $\alpha \in \pi_{*}^{s}\left(S^{0}\right)$ by $e(\alpha)$, and refer to [1] for its various properties.

(1.5) For the stunted projective spaces, $p_{k, l}: H P_{k}^{n} \rightarrow H P_{l}^{n}$ and $i_{k, l}: H P_{m}^{k} \rightarrow H P_{m}^{l}$ $(1 \leqq m \leqq k \leqq l \leqq n \leqq c \odot)$ denote the collapsing map and the inclusion map respectively. Also $\partial_{k}: H P_{k+1}^{n} \rightarrow \Sigma H P^{k}(n \geqq k)$ denotes the map which appears in the cofiber sequence as follows:

$$
H P^{k} \stackrel{i_{k, n}}{\longrightarrow} H P^{n} \stackrel{p_{1, k+1}}{\longrightarrow} H P_{k+1}^{n} \stackrel{\partial_{k}}{\longrightarrow} \Sigma H P^{k} .
$$

For the stunted complex projective spaces, we use the similar notations.

This paper is organized as follows:

In Section 2, we prepare a stable map between the quaternionic projective spaces, 
and state Theorem 3 which is essential in the proof of Theorem 5. In Section 3 we state and prove the main theorem (Theorem 5), and Sections 4 and 6 are devoted to the proofs of Theorems $\mathrm{A}$ and $\mathrm{B}$ respectively. In Section 5 , we prove Theorem 3 .

The authors thank $H$. Oshima for his valuable informations on the James numbers.

\section{§. The Stable Map $f$}

In $[7]$, the second named author showed the existence of some stable maps $\Sigma^{n} H P^{\circ} \rightarrow H P^{\infty}$. Using its result, we have the following

Theorem 1. ([7; Theorem 1]) There is a stable map

$$
f: \Sigma^{\circledR} H P^{\infty} \longrightarrow H P^{\infty}
$$

which satisties

$$
f_{*}\left(\beta_{n}\right)=((2 n+4) ! /(2 n) !) \beta_{n+2} \quad \text { for } n \geqq 1 \text {. }
$$

Consider the restriction of $f$ to $\Sigma^{s} H P^{n}$ for some $n$. Then, by the cellular approximation, the range of $f$ can be taken as $H P^{n+2}$, that is, we have a map $\Sigma^{8} H P^{n} \rightarrow H P^{n+2}$. Especially, let

$$
f_{1}: S^{12}=\Sigma^{8} H P^{1} \longrightarrow H P^{3}
$$

be the restriction to $\Sigma^{8} H P^{1}$. Then, by Theorem 1 , we have

$$
f_{1 *}\left(\beta_{1}\right)=360 \beta_{3} .
$$

Since $H P_{2}^{3}$ is the mapping cone of $2 j_{3}$ and the order of $j_{3}$ is equal to 21 , there is a unique map

$$
g: S^{12} \longrightarrow H P_{2}^{3} \text { satisfying } g_{:}\left(r_{12}\right)=12 \beta_{3} .
$$

where $\iota_{12} \in H_{12}\left(S^{12}\right)$ is a generator.

By an easy computation, we have the following lemma, which is an immediate consequence of [5] or [6] for the 2-localized case. In its equality, $\partial_{1}: H P_{2}^{3} \rightarrow$ $\Sigma H P^{1}=S^{5}$ denotes the map mentioned in (1.5).

$$
\text { Lemma 2. } \partial_{1} g=8 j_{7} .
$$

Let $M_{t}$ be the mod $t$ Moore spectrum $S^{0} \bigcup_{t} e^{1}$, and $i_{0}: S^{n} \rightarrow I_{i}$ and $p_{1}: M_{t} \rightarrow$ $S^{1}$ be the inclusion and the projection respectively.

By (2.2) and (2.3), we have $p_{1,2} f_{1}=30 g$, where $p_{1,2}: H P^{:} \rightarrow H P_{2}^{3}$ is the collapsing map. Hence, there is a map $h(1): \Sigma^{7} M_{30} \rightarrow S^{0}$ such that the following diagram is commutative up to sign: 


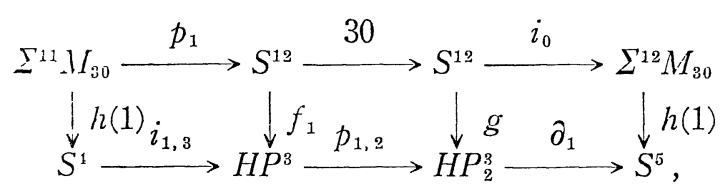

where horizontal sequences are cofiberings. Then, by Lemma 2 and (2.4), it follows that

$$
h(1) \text { is an extension of } 8 j_{7} .
$$

Also the order of $h(1)$ is a divisor of 30 , because, by (2.5) and [12; Proposition $1.9,(3.10)], 30 h^{\prime}(1) \equiv p_{\mathrm{I}}^{*}\left\langle 30,8 j_{7}, 30\right\rangle=0$. Thus, there is a coextension $A(1): \Sigma^{8} M_{30}$ $\rightarrow M_{30}$ of $h(1)$. Then $A(1)$ satisfies $p_{1} A(1) i_{0}=8 j_{7}$.

Since the order of $j_{3}$ is equal to 24 , we have an extension $h(2): \Sigma^{3} M_{24} \rightarrow S^{0}$ of $j_{3}$. On the other hand, since $\left\langle 24,10 j_{7}, 24\right\rangle=0$ by $[12 ;(3.10)]$, there is a map $A(2): \Sigma^{8} M_{24} \rightarrow M_{21}$ which satisfies $p_{1} A(2) i_{0}=10 j_{7}$.

Now, let $M(\varepsilon)=M_{30}$ if $\varepsilon=1$ and $M(\varepsilon)=M_{21}$ if $\varepsilon=2$. Then, we have the following

Theorem 3. Let $\varepsilon=1$ or 2 , and let $k(\varepsilon)=7$ (resp. 3) if $\varepsilon=1$ (resp. 2). Then the following dingram is commutative:

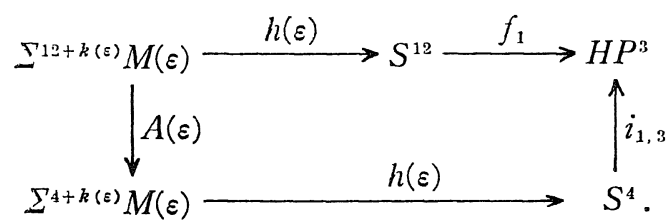

We prove this theorem in Section 5, and in Sections 3 and 4, we assume it.

\section{§3. The Main Theorem}

Let $M\left(\varepsilon, h \xi^{\prime}\right.$ and $A(\varepsilon)(\varepsilon=1,2)$ be the spaces and maps defined in the previous section.

Now, we denine a class $\alpha(n) \in \pi_{\sharp n-\jmath}^{s}\left(S^{0}\right)(n \geqq 2)$ as follows :

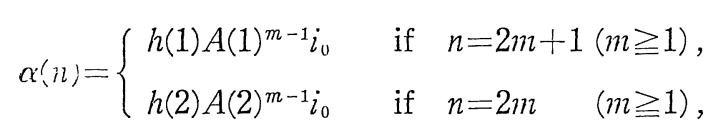

where $i_{0}: S^{r} \rightarrow \Sigma^{t} M(\xi)$ are the respective inclusions. Note that $\alpha(2)=j_{3}$ and $\alpha(3)=8 j_{7}$. Then, from this definition, the following proposition follows immediately, but we shall not use it in this paper:

Proposition 4. Let $m \geqq 1$.

(i) The order of $\alpha(2 m+1)$ is equal to 30 , and

$$
\alpha(2 m+3) \in\left\langle 8 j_{7}, 30, \alpha(2 m+1)\right\rangle .
$$


(ii) The order of $\alpha(2 m)$ is equal to 24 , and

$$
\alpha(2 m+2) \in\left\langle\alpha(2 m), 24,10 j_{7}\right\rangle .
$$

Consider the following Atiyah-Hirzebruch spectral sequence:

$$
E_{p, q}^{2}=\tilde{H}_{p}\left(H P^{\infty}\right) \otimes \pi_{q}^{s}\left(S^{0}\right) \Longrightarrow \pi_{p+q}^{s}\left(H P^{\infty}\right),
$$

and let $d^{r}: E_{p, q}^{r} \rightarrow E_{p-r, q+r-1}^{r}(r \geqq 2)$ be the differential in this spectral sequence. If an element $\gamma \in E_{p, q}^{2}$ persists to $E_{p, q}^{r}$, then we denote its class in $E_{p, q}^{r}$ simply by $\gamma$.

Let $t_{n}(n \geqq 2)$ be the following integer :

$$
t_{n}=(2 n) ! / 60 \text { if } n \text { is odd, and } t_{n}=(2 n) ! / 24 \text { if } n \text { is even . }
$$

Now, we can state the main theorem as follows:

Theorem 5. For $n \geqq 2$,

$$
t_{n} \beta_{n} \in E_{4 n, 0}^{4 n-4} \text { and } d^{4 n-1}\left(t_{n} \beta_{n}\right)=\beta_{1} \otimes \alpha(n) .
$$

The rest of this section is devoted to the proof of Theorem 5 .

First, we prepare the following diagram $(n \geqq 2)$ :

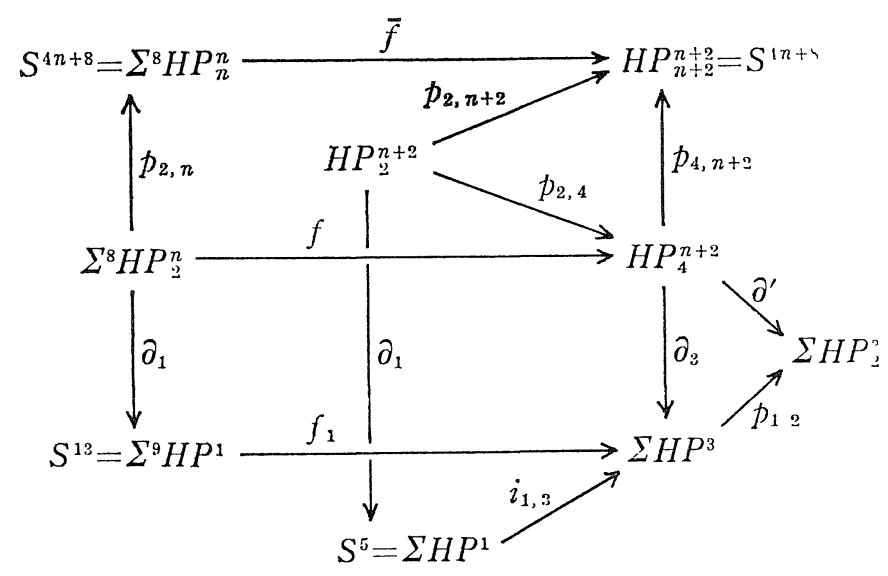

Here, the maps $f$ and $\bar{f}$ are defined from the map of Theorem 1 by restricting to $\Sigma^{8} H P^{k}(k=1, n-1, n), f_{1}$ is the map in (2.1), the maps $i_{k, l}, p_{k, l}$ and $\partial_{k}$ are mentioned in (1.5), and $\partial^{\prime}=p_{1,2} \partial_{3}$. Then the squares and the triangles in (3.4) are commutative, and the two sequences

$$
H P_{2}^{n+2} \stackrel{p_{2,4}}{\longrightarrow} H P_{4}^{n+2} \stackrel{\partial^{\prime}}{\longrightarrow} \Sigma H P_{2}^{3} \text { and } \Sigma H P^{1} \stackrel{i_{1,3}}{\longrightarrow} \Sigma H P^{3} \stackrel{p_{1,2}}{\longrightarrow} \Sigma H P_{2}^{3}
$$

in (3.4) are cofiberings.

We prove Theorem 5 by induction on $n$. 
When $n=2$, the theorem is clear, since $H P^{2}$ is the mapping cone of $j_{3}$. Also, when $n=3$, the theorem follows from Lemma 2 .

By the definition of $d^{r}$ in the spectral sequence (3.2), the assertion that Theorem 5 holds for $n$ is equivalent to that there is a map

$$
X(n): S^{4 n} \longrightarrow H P_{2}^{n}
$$

which satisfies that

$$
h_{n, 2}(X(n))=t_{n} \beta_{n}
$$

and

$$
\partial_{1} X(n)=\alpha(n),
$$

where $h_{n, 2}$ is the stable Hurewicz homomorphism (see (1.1)). Therefore, by induction, we may assume the existence of such a map $X(n)$ in $(3.5)_{n}$ for $n \geqq 2$ that satisfies the properties in $(3.6)_{n}$ and $(3.7)_{n}$, and under these assumptions it is enough to prove that the assertion also holds for $n+2$, that is, there exists a map $X(n+2)$ in (3.5) $)_{n+2}$ satisfying (3.6) $)_{n+2}$ and $(3.7)_{n+2}$.

By Theorem 3 and the definition of $\alpha(n)$ in (3.1), we have

$$
f_{1} \alpha(n)=i_{1,3} \alpha(n+2) \text {. }
$$

Then, using (3.7) ${ }_{n}$ and the commutativity of (3.4), we have $\partial^{\prime} f X(n)=0$. Hence, there is a map $X^{\prime}: S^{4 n+8} \rightarrow H P_{2}^{n+2}$ such that $p_{2,4} X^{\prime}=f X(n)$. Then, by (3.8) and (3.4), we have $i_{1,3} \partial_{1} X^{\prime}=\partial_{3} f X(n)=f_{1} \alpha(n)=i_{1,3} \alpha(n+2)$. Thus $\partial_{1} X^{\prime}-\alpha(n+2) \in \operatorname{Ker} i_{1,3^{*}}$, and so there is a map $y^{\prime}: S^{4 n+8} \rightarrow H P_{2}^{3}$ which satisfies that

$$
\partial_{1}\left(X^{\prime}+i_{3, n+2} y^{\prime}\right)=\alpha(n+2) .
$$

Now we define a map $X(n+2)$ by

$$
X(n+2)=X^{\prime}+i_{3, n+2} y^{\prime} .
$$

Then, (3.9) yields that

$$
\partial_{1} X(n+2)=\alpha(n+2) .
$$

On the other hand, $p_{2, n+2} i_{3, n+2} y^{\prime}=0$, since $i_{3, n+2} y^{\prime}$ is a torsion element in $\pi_{4 n+8}^{s}\left(H P_{2}^{n+2}\right)$. Hence, we have

$$
p_{2, n+2} X(n+2)=p_{2, n+2} X^{\prime}=\bar{f} p_{2, n} X(n) .
$$

This implies that $h_{n+2,2}(X(n+2))=\bar{f}_{*} h_{n, 2}(X(n))=f_{*} h_{n, 2}(X(n))$. But, by Theorem 1 and the assumption $(3.6)_{n}$, we have $f_{*} h_{n, 2}(X(n))=((2 n+4) ! /(2 n) !) t_{n} \beta_{n+2}=t_{n+2} \beta_{n+2}$. Thus

$$
h_{n+2,2}(X(n+2))=t_{n+2} \beta_{n+2},
$$

and we have completed the proof of Theorem 5 . 


\section{$\S 4$. Proof of Theorem A}

We use the following notations: Let $k, l$ be any integers. Then $k \mid l$ means that $k$ is a divisor of $l, \nu_{2}(k)$ is the exponent of 2 in the prime power decomposition of $k$, and

$$
a(k)=1 \text { if } k \text { is even and } a(k)=2 \text { if } k \text { is odd. }
$$

Recall that $\left|h_{n, 2}\right|$ is the order of the cokernel of the stable Hurewicz homomorphism $h_{n, 2}$ (see (1.1)). Then, Theorem 5 yields that

$$
\left|h_{n, 2}\right| \mid t_{n} \text {. }
$$

Hence, we have

$$
\nu_{2}\left(\left|h_{n, 2}\right|\right) \leqq \nu_{2}\left(t_{n}\right)=\nu_{2}(a(n)((2 n) !) / 8) .
$$

Thus, in order to complete the proof of Theorem A, it is sufficient to show the following proposition.

Proposition 6. $\nu_{2}(a(n)((2 n) !) / 8) \leqq \nu_{2}\left(\left|h_{n, 2}\right|\right) \quad$ for $n \geqq 2$.

We shall prove this proposition by using standard arguments of $K$-theory and Chern character. For this, we prepare some notations.

Let $K, K O$ and $K S p$ denote the complex, real and symplectic $K$-theory respectively, and $K^{*}(X)$ and $K O^{*}(X)$ denote the $K$ - and $K O$-cohomology respectively. Let $\xi$ be the canonical quaternionic line bundle over $H P^{n}(1 \leqq n \leqq \infty)$, and $\tilde{\xi}=\xi-1$ $\in \widetilde{K S} p\left(H P^{n}\right)=\widetilde{K O^{4}}\left(H P^{n}\right)$. Then, as is well known, $p_{1, k}^{*}: \widetilde{K O^{*}}\left(H P_{k}^{n}\right) \rightarrow \widetilde{K O} *\left(H P^{n}\right)$ is monomorphic and $\widetilde{K O} *\left(H P_{k}^{n}\right)$ is a free module over $\pi_{*}(K O)$ with basis $\{\xi(s)$ : $k \leqq s \leqq n\}$ whose element $\xi(s)$ satisfies $p_{1, k}^{*}(\xi(s))=\tilde{\xi}^{s}$.

Let $c: \widetilde{K O} *(X) \rightarrow \widetilde{K}^{*}(X)$ be the complexification, and $c h: \tilde{K}^{*}(X) \rightarrow \widetilde{H}^{*}(X ; Q)$ be the Chern character. The composition $c h{ }^{\circ} \mathrm{c}$ is called the Pontrjagin character and we denote it by $p h: \widetilde{K O^{*}}(X) \rightarrow \widetilde{H}^{*}(X ; Q)$.

Let $x \in H^{4}\left(H P^{n}\right)$ be the Euler class of $\xi$. Then $H^{*}\left(H P^{n}\right)=Z[x] /\left(x^{n+1}\right)$. We use the same letter $x^{i}$ to denote the element of $H^{i i}\left(H P_{k}^{n}\right)$. Then it is vell known that

$$
\operatorname{ph}(\xi(s))=\left(e^{-\bar{x}}+e^{-{ }^{\prime} \bar{x}}-2\right)^{s}=\left(\sum_{j=1}(2 /(2 j) !) x^{-j}\right)^{s} .
$$

Let $N(m, s)$ be the coefficient of $x^{m}$ in (4.4), that is,

$$
\sum_{m=1} N(m, s) x^{m}=\left(\sum_{j=1}(2 /(2 j) !) x^{j}\right)^{s} .
$$

Then we have

Lemma 7. For $k \leqq s$,

$$
\left|h_{n, k}\right| N(n, s) \in a(n-s) Z .
$$


Proof. Let $\alpha \in \pi_{+n}^{s}\left(H P_{k}^{\infty}\right)$ be a class such that $h_{n, k}(\alpha)=\left|h_{n, k}\right| \beta_{n}$. We can consider $\alpha$ to be a map $S^{4 n} \rightarrow H P_{k}^{n}$ by the cellular approximation. Then, by definition, we have

$$
\alpha^{*}\left(x^{n}\right)=\left|h_{n, k}\right| \iota_{1 n} \text { for a generator } \iota_{4 n} \in H^{1 n}\left(S^{4 n} ; Z\right) .
$$

By (4.4), (4.5) and (4.6), we have

$$
p h\left(\alpha^{*} \xi(s)\right)=\alpha^{*}\left(N(n, s) x^{n}\right)=\left|h_{n, k}\right| N(n, s) \iota_{1 n} .
$$

On the other hand, as is well known, both $\widetilde{K}^{4 s}\left(S^{4 n}\right)$ and $\widetilde{K O^{1 s}(}\left(S^{4 n}\right)$ are isomorphic to $Z$, and the complexification $c: \widetilde{K O^{1 s}}\left(S^{4 n}\right) \rightarrow \widetilde{K}^{4 s}\left(S^{1 n}\right)$ sends the generator of $\widetilde{K O^{4 s}}\left(S^{4 n}\right)$ to the $a(n-s)$ times of the one of $\widetilde{K}^{t s}\left(S^{4 n}\right)$. Moreover the Chern character gives an isomorphism $\widetilde{K}^{1 s}\left(S^{4 n}\right) \rightarrow \widetilde{H}^{*}\left(S^{4 n} ; Z\right)$. Since $\alpha^{*} \xi(s) \in \widetilde{K O^{4 s}}\left(S^{4 n}\right)$, it follows that

$$
\operatorname{ph}\left(\alpha^{*} \xi(s)\right) \in a(n-s) H^{4 n}\left(S^{4 n} ; Z\right),
$$

and the result follows from (4.7) and (4.8).

q.e.d.

Proof of Proposition 6. By an elementary arithmetic, we have that $N(n, 2)$ $=8\left(4^{n-1}-1\right) /(2 n)$ !. Hence, by Lemma $7,8\left(4^{n-1}-1\right)\left|h_{n, 2}\right| /(a(n)((2 n) !))$ is an integer. Since $a(n)((2 n) !) / 8$ is an integer for $n \geqq 2$, we have the desired result. q.e.d.

\section{\$5. Proof of Theorem 3}

Proof of Theorem 3 for $\varepsilon=1$. Using the commutativity of the left and the right squares of (2.4) and by that $A(1)$ is a coextension of $h(1)$, we have

$$
f_{1} h(1)=f_{1} p_{1} A(1)=i_{1,3} h(1) A(1),
$$

and the theorem holds in this case.

Proof of Theorem 3 for $\varepsilon=2$. Recall that $M(2)=M_{24}$ is the $\bmod 24$ Moore spectrum, $h(2)$ is any extension of $j_{3}$ and $A(2)$ is a map which satisfies $p_{1} A(2) i_{0}$ $=10 j_{7}$. In the following diagram, we explain notations which we shall use in the proof:

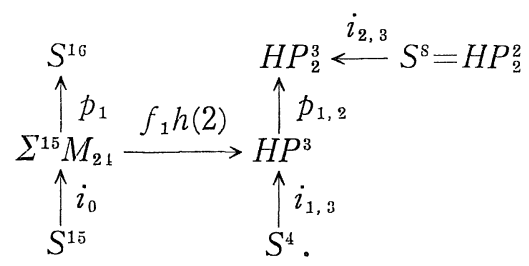

Lemma 8. The composition $p_{1,2} f_{1} h(2): \Sigma^{15} M_{21} \rightarrow H P_{2}^{3}$ is null homotopic.

Proof. We remark that $H P_{2}^{3}$ is a mapping cone of $2 j_{3}$ and that $f_{1^{*}}\left(\beta_{1}\right)=$ 
$360 \beta_{3}$ by (2.2). Then, using [12; Proposition 1.8] and [1; Theorem 11.1], we have

$$
p_{1,2} f_{1} h(2) i_{0} \in\left(i_{2,3}\right)_{*}\left\langle 2 j_{3}, 360, j_{3}\right\rangle=0 .
$$

Thus there is a class $\alpha \in \pi_{16}^{s}\left(H P_{2}^{3}\right)$ such that

$$
p_{1,2} f_{1} h(2)=\alpha p_{1}
$$

Now, we show that

$$
\alpha=0 \text {. }
$$

Then, by (5.2), we have the lemma.

Let $\eta \in \pi_{1}^{s}\left(S^{0}\right)$ be a generator. Then there is an extension $\bar{\gamma}_{1}: H P_{2}^{3} \rightarrow S^{7}$ of $r_{l}: S^{8} \rightarrow S^{7}$, and we have

$$
\bar{\eta} p_{1,2} f_{1} h(2)=0,
$$

because $\bar{\eta} p_{1,2} f_{1} \in \pi_{5}^{s}\left(S^{0}\right)=0$. Therefore, $\bar{\eta} \alpha p_{1}=0$. Since $\pi_{4}^{s}\left(S^{0}\right)=0$, $\alpha$ factors a map $\alpha_{1}: S^{16} \rightarrow S^{8}=H P_{2}^{2}$, and we have

$$
\eta \alpha_{1} p_{1}=0 \text {. }
$$

But $\eta^{*}: \pi_{8}^{s}\left(S^{0}\right) \rightarrow \pi_{9}^{s}\left(S^{0}\right)$ is monomorphic by the table of [12; Chapter XIV], and $p_{1}^{*}:\left\{S^{16}, S^{7}\right\} \rightarrow\left\{\Sigma^{15} M_{21}, S^{7}\right\}$ is monomorphic by that $2 \pi_{9}^{8}\left(S^{0}\right)=0$. Thus $\alpha_{1}=0$ by (5.5), and we have (5.3).

q.e.d.

By the above lemma, there is a map $\varphi: \Sigma^{15} M_{21} \rightarrow S^{4}$ which satisfies

$$
i_{1,3} \varphi=f_{1} h(2) .
$$

Lemma 9. $e\left(\varphi i_{0}\right)=1 / 24$,

where $e\left(\varphi i_{0}\right)$ is the Adams $e_{R}{ }^{\prime}$-invariant of $\varphi i_{v}$.

Proof. Recall that $p h: \widetilde{K O}() \rightarrow \widetilde{H}^{*}(; Q)$ is the Pontrjagin character and $\tilde{\xi} \equiv \widetilde{K O}\left(\Sigma^{4} H P^{3}\right)$ is the element corresponding to $\xi-1 \in \widetilde{K S} p\left(H P^{3}\right)$ under the Bott isomorphism (see Section 4). We denote the standard generator of $\widetilde{K O}\left(S^{s i}\right)$ by $g_{\mathrm{s} \iota}$. As is well-known (cf. [1], [13]), Adams $e_{R}{ }^{\prime}$-invariant is a functional Pontrjagin character. In our case we have

$$
e\left(\varphi i_{0}\right)=\left(p h_{20}\right)_{\varphi \iota_{0}}\left(g_{s}\right),
$$

where $p h_{20}$ is the 20-dimensional component of $p h$ and $\left(p h_{20}\right)_{\varphi i_{0}}: \widetilde{K O}\left(S^{\diamond}\right) \rightarrow$ $\tilde{H}^{*}\left(S^{20} ; Q\right) / \operatorname{Im} p h_{20} \cong Q / Z$ is the functional Pontrjagin character of $\varphi i_{0}$. We put $\zeta=\varphi i_{0}$. By (5.6) we have the following commutative diagram:

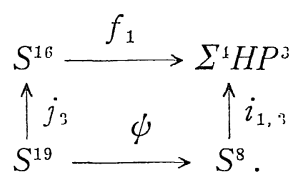


Then the following diagram is commutative:

$$
\begin{aligned}
& \widetilde{K O}\left(\sum^{4} H P^{3}\right) \stackrel{i_{1,3}^{*}}{\longrightarrow} \widetilde{K O}\left(S^{s}\right) \\
& \quad \downarrow f_{1}^{*} \\
& \widetilde{K O}\left(S^{16}\right) \stackrel{\left(p h_{20}\right)_{j_{3}}}{\longrightarrow} H^{20}\left(S^{20} ; Q\right) / \operatorname{Im} p h_{20} .
\end{aligned}
$$

It is clear that $i_{1}^{*},{ }_{3} \tilde{\xi}=g_{8}$. On the other hand,

$$
f_{1}^{*} \tilde{\xi}=g_{16} .
$$

In fact, $f_{1}^{*}\left(x^{-3}\right)=360 \iota_{16}$ by $(2.2)$, and so $p h\left(f_{1}^{*} \tilde{\xi}\right)=f_{1}^{*}\left((2 /(6 !)) x^{3}\right)=\iota_{16}$, where $x \subseteq$ $H^{4}\left(H P^{3} ; Z\right) \subset H^{4}\left(H P^{3} ; Q\right)$ is the Euler class of $\xi$. Since $p h: \widetilde{K O}\left(S^{16}\right) \rightarrow H^{16}\left(S^{16} ; Z\right)$ is isomorphic, we have (5.9).

Now, by the naturality of the functional operation, (5.8) and the above equalities, we have

$$
\begin{aligned}
\left(p h_{20}\right)_{\dot{\varphi}}\left(g_{8}\right) & =\left(p h_{20}\right)_{\dot{\varphi}}\left(i_{1,3}^{*} \tilde{\xi}\right)=\left(p h_{20}\right)_{j_{3}}\left(f_{1}^{*} \tilde{\tilde{\xi}}\right) \\
& =\left(p h_{20}\right)_{j_{3}}\left(g_{16}\right) .
\end{aligned}
$$

But, $\left(p h_{20}\right)_{J_{3}}\left(g_{16}\right)=e\left(j_{3}\right)=1 / 24$. Thus (5.7) and (5.10) give the desired result.

q.e.d.

Now, $\pi_{11}^{s}\left(S^{0}\right)=\operatorname{Im} J_{11}$ and the order of $\pi_{11}^{s}\left(S^{0}\right)$ is equal to 504 (cf. [1; Example 7.17]). Hence by the above lemma and [1], we have

$$
\varphi i_{0}=21 j_{11} \text {. }
$$

On the other hand, using [12; Proposition 1.7] and [1; Theorem 11.1], we have

$$
h(2) A(2) i_{0} \in\left\langle j_{3}, 24,10 j_{7}\right\rangle=21 j_{11} .
$$

But $i_{0}^{*}:\left\{\Sigma^{11} M_{24}, S^{0}\right\} \rightarrow \pi_{11}^{s}\left(S^{0}\right)$ is monomorphic, because $\pi_{12}^{s}\left(S^{0}\right)=0$ (cf. [12]). Hence (5.11) and (5.12) yield that

$$
\varphi=h(2) A(2) .
$$

Thus, by (5.6) and (5.13), we have

$$
i_{1,3} h(2), A(2)=f_{1} h(2),
$$

and this completes the proof of Theorem 3 .

\section{$\S 6$. Proof of Theorem B}

For the homology of the complex projective space, we denote the standard generators by $b_{i} \in H_{2 i}\left(C P^{m} ; Z\right)(1 \leqq i \leqq n \leqq \infty)$. Then, $\tilde{H}_{*}\left(C P_{k}^{m} ; Z\right)$ is a free abelian group with basis $\left\{b_{k}, \cdots, b_{m}\right\}$.

By the definition of $U(n, k)$ in Section 1 , we have that $U(n, k)$ is the stable 
order of the attaching map of the top cell in $C P_{n-k}^{n-1}$, or equivalently,

$$
\operatorname{Im} h(n-1, n-k) \text { is generated by } U(n, k) b_{n-1} \text {, }
$$

where

$$
h(m, l): \pi_{2 m}^{s}\left(C P_{l}^{m}\right) \longrightarrow H_{2 m}\left(C P_{\iota}^{\prime n} ; Z\right)
$$

is the stable Hurewicz homomorphism.

Now using $K$-theory for $C P_{l}^{m}$ and $S^{2 m}(m=n-1, l=n-k)$ just in the same way as Lemma 7 , we have immediately the following lemma, where $B(n, s)$ denotes the rational number which is the coefficient of $y^{n-1}$ in the formal power series $\left(e^{y}-1\right)^{s} \quad(s \geqq 1)$ :

Lemma 10. The number $U(n, n-k) B(n, s)$ is an integer for $k \leqq s$.

By an elementary arithmetic, we have that $B(2 n+1,3)=3\left(3^{2 n-1}+1-2^{2 n}\right) /(2 n)$ ? and $\nu_{2}\left(3^{2 n-1}+1-2^{2 n}\right)=2$ for $n \geqq 2$. Thus we have

Corollary 11. $\nu_{2}((2 n) ! / 4) \leqq \nu_{2}(U(2 n+1,2 n-2)) \quad$ for $n \geqq 2$.

Remark. The lower estimation of $U(m, l)$ is studied by G. Walker [13] by using $K O$-theory, and Lemma 10 may be a weaker condition than his result. But, for our restricted purpose, Lemma 10 is sufficient.

Let $S^{2} \rightarrow C P^{\infty} \stackrel{q}{\rightarrow} H P^{\mathrm{c}}$ be the usual fibration, and let

$$
t: H P_{+}^{\infty} \longrightarrow C P_{+}^{\infty}
$$

be the Becker-Gottlieb transfer [2] for this fibration. By the cellular approximation, a map

$$
t: H P_{2}^{n} \longrightarrow C P_{3}^{2 n} \quad \text { for } n \geqq 2
$$

is induced from (6.3). Recall that the Euler characteristic of $S^{2}$ is equal to 2 . Hence according to $\left[2\right.$; Theorem 5.5], we have $q_{*} t_{*}\left(\beta_{n}\right)=2 \beta_{n}$ for the standard generator $\beta_{n} \in H_{4 n}\left(H P^{\infty}\right)$. Since $q_{*}: H_{4 n}\left(C P^{\infty}\right) \rightarrow H_{1 n}\left(H P^{\infty}\right)$ is isomorphic, we have

$$
t_{*}\left(\beta_{n}\right)=2 b_{2 n} \text {. }
$$

Let $X(n) \in \pi_{4 n}^{s}\left(H P_{2}^{n}\right)$ be the class of $(3.5)_{n}$. Then, by $(3.6)_{n}$ and (6.5), we have

Lemma 12. $h(2 n, 3)\left(t_{*}(X(n))\right)=2 t_{n} b_{2 n}$, where $t_{n}$ is the integer in (3.3).

By (6.1) this lemma implies that the number $U(2 n+1,2 n-2)$ is a divisor of $2 t_{n}$. Thus we have

Corollary 13. $\nu_{2}(U(2 n+1,2 n-2)) \leqq \nu_{2}(a(n)((2 n) ! / 4))$, where $a(n)=1$ if $n$ is ce'en and $a(n)=2$ if $n$ is odd. 
For even $n \geqq 2$, we have completed the proof of Theorem B by Corollaries 11 and 13.

To prove Theorem B for odd $n$, we need some notations.

According to [11], there is a stable map

$$
F: \Sigma^{2} C P^{\infty} \longrightarrow C P^{\infty}
$$

which satisfies

$$
F_{*}\left(b_{n}\right)=(n+1) b_{n+1} \quad \text { for } \quad n \geqq 1 .
$$

Let

$$
F_{2}: \sum^{4} C P^{2} \longrightarrow C P^{4}
$$

be the restriction of $F \circ F: \Sigma^{4} C P^{\infty} \rightarrow C P^{\infty}$ to $\Sigma^{4} C P^{2}$. Then from (6.7), it follows that

$$
F_{2^{*}}\left(b_{n}\right)=(n+1)(n+2) b_{n+2}(n \geqq 1) .
$$

Now we consider the composition

$$
G=p_{1,3} \circ F_{2} \circ t: S^{8}=\Sigma^{4} H P^{1} \longrightarrow \Sigma^{ \pm} C P^{2} \longrightarrow C P^{1} \longrightarrow C P_{3}^{4},
$$

where $t: H P^{1} \rightarrow C P^{2}$ is the restriction of the transfer of (6.3). Then we have

Lemma 14. The composition $G \circ h(2): \Sigma^{11} M_{24} \rightarrow C P_{3}^{4}$ is null homotopic, where $h(2): \Sigma^{11} M_{24} \rightarrow S^{8}$ is an extension of $j_{3}$ (see Section 2).

Proof. In the following diagram, we explain notations used in this proof:

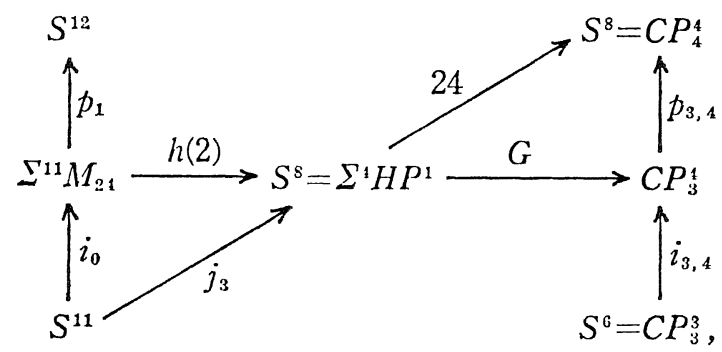

where $i_{0}$ and $i_{3,4}$ are the respective inclusions and $p_{1}$ and $p_{3,4}$ are the respective projections.

Now, by $(6.5)$ and (6.9), we have $G_{*}\left(b_{1}\right)=24 b_{\star}$. Hence, by using [12 ; Proposition $1.9,(3.10)]$, we have

$$
p_{3,4}{ }^{\circ} G \circ h(2) \in p_{1}^{*}\left\langle 24, j_{3}, 24\right\rangle=0 .
$$

Thus, there is a map $\bar{\alpha}: \sum^{11} M_{24} \rightarrow S^{6}$ such that $G \circ h(2)=i_{3,4} \bar{\alpha}$. Moreover, since $\pi_{\overline{5}}^{s}\left(S^{0}\right)=0, \bar{\alpha}$ factors $\alpha: S^{12} \rightarrow S^{6}$, that is, $G \circ h(2)=i_{3,1} \alpha p_{1}$. Hence it is sufficient to show that 


$$
\alpha=0 \text { 。 }
$$

We remark that

(6.13) $\pi_{6}^{s}\left(S^{0}\right)$ is a group of order 2 and generated by $\left(j_{3}\right)^{2}$ (cf. [12]).

Hence $\alpha=0$ or $\left(j_{3}\right)^{2}$. Now we suppose that $\alpha=\left(j_{3}\right)^{2}$. Then $j_{3} \alpha=\left(j_{3}\right)^{3} \neq 0$ (cf. [12]), and

$$
j_{3} \alpha p_{1} \neq 0,
$$

because the homomorphism $p_{1}^{*}: \pi_{12}^{s}\left(S^{6}\right) \rightarrow\left\{\Sigma^{11} M_{24}, S^{6}\right\}$ is monomorphic by (6.13). However, since $C P_{3}^{4}$ is a mapping cone of a generator $\eta$ of $\pi_{1}^{s}\left(S^{0}\right)$ and since $j_{3} \eta=0$, there is an extension $\bar{j}_{3}: C P_{3}^{4} \rightarrow S^{3}$ of $j_{3}: C P_{3}^{3}=S^{6} \rightarrow S^{3}$. But $\bar{j}_{3} G \in \pi_{8}^{s}\left(S^{3}\right)$ $=0$, and so $j_{3} \alpha p_{1}=\bar{j}_{3} G h(2)=0$. This contradicts (6.14), and we have (6.12).

q.e.d.

Let $Y(2 m)$ be the composition $t \circ X(2 m): S^{8 m+1} \rightarrow \Sigma^{4} H P_{2}^{2 m} \rightarrow \Sigma^{4} C P_{3}^{4 m}$ (see the definition of $X(2 m)$ to (3.5)). Then we have the following commutative diagram:

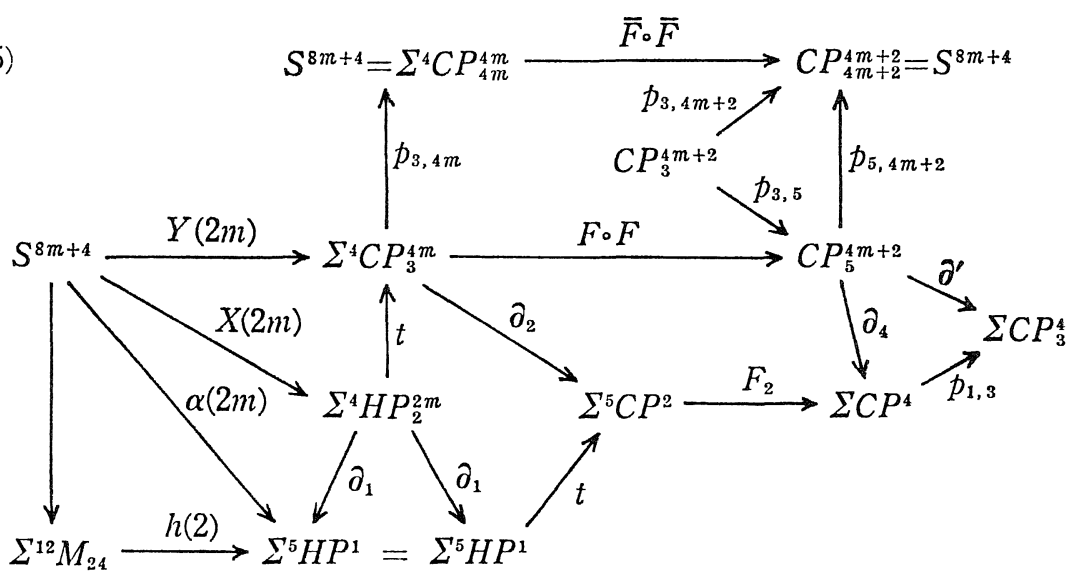

where the maps $F$ and $\bar{F}$ are defined from the map in (6.6) by restricting it, $F_{2}$ is the map in (6.8), and $t$ are the maps in (6.4) and (6.10)

By Lemma 14, we have

$$
p_{1,} F_{2} t \alpha(2 m)=0 \text {. }
$$

Moreover, the sequence

$$
C P_{3}^{1 m+2} \stackrel{p_{3,5}}{\longrightarrow} C P_{5}^{+m+2} \stackrel{\partial^{\prime}}{\longrightarrow} \Sigma C P_{3}^{\vdots}
$$

in (6.15) is a cofibering. Therefore, by chasing the diagram (6.15), we have a map

$$
Y(2 m+1): S^{s m+4} \longrightarrow C P_{3}^{4 m+2}
$$


which satisfies

$$
p_{3,5} Y(2 m+1)=(F \circ F) Y(2 m) \text {. }
$$

Then it follows that

$$
p_{3,4 m+2} Y(2 m+1)=(\bar{F} \circ \bar{F}) p_{3,1 m} Y(2 m) \text {. }
$$

By (3.6) $)_{2 m}$ and (6.5), $Y(2 m)_{*}\left(\iota_{8 m+4}\right)=t_{*} X(2 m)_{*}\left(\iota_{8 m+4}\right)=2 t_{2 m} b_{4 m}$ for a generator $\iota_{l} \in$ $H_{l}\left(S^{l} ; Z\right) \quad(l=8 m+4)$. Also by $(6.7),(\bar{F} \circ \bar{F})_{*}\left(b_{4 m}\right)=(F \circ F)_{*}\left(b_{4 m}\right)=(4 m+2)(4 m+1)$ $b_{i m+2}$. Thus these equalities and (6.18) give

$$
h(2 n, 3)(Y(n))=((2 n) ! / 12) b_{n} \quad(n=2 m+1) .
$$

By (6.1), this implies that

$$
U(2 n+1,2 n-2) \mid((2 n) ! / 12) \quad(n=2 m+1),
$$

and we have completed the proof of Theorem B by Corollary 11 and (6.20).

\section{References}

[1] Adams, J.F., On the groups $J(X)$-IV, Topology, 5 (1966), 21-71.

[2] Becker, J.C. and Gottlieb, D. H., The transfer map and fiber bundles, Topology, 14 (1975), 1-12.

[3] James, I. M., Spaces associated with Stiefel manifolds, Proc. London Math. Soc., (3) 9 (1959), 115-140.

[4] Knapp, K., Some applications of $K$-theory to framed bordism : $E$-invariant and transfer, Habilitationsschrift, Bonn, 1979.

[5] Kochman, S. O. and Snaith, V.P., On the stable homotopy of symplectic classifying and Thom spaces, Lectures Notes in Math., 741, Springer-Verlag, 1979, 394-448.

[6] Morisugi, K., Massey products in $\mathrm{MSp}_{*}$ and its application, J. Math. Kyoto Univ., 23 (1983), 241-265.

[7] - Stable self maps of the quaternionic (quasi-) projective space, preprint.

[8] Ōshima, H., On James numbers of stunted complex or quaternionic projective spaces, Osaka J., Math., 16 (1979), 479-504.

[9] - Some James numbers of Stiefel manifolds, Math. Proc. Camb. Phil. Soc., 92 (1982), 139-161.

[10] Segal, D. M., On the stable homotopy of quaternionic and complex projective spaces, Proc. Amer. Math. Soc., 25 (1970), 838-841.

[11] Toda, H., A topological proof of theorems of Bott and Borel-Hirzebruch for homotopy groups of unitary groups, Memoirs Univ. of Kyoto, 32 (1957), 103-119.

[12] - Composition methods in homotopy groups of spheres, Annals of Math. Studies, 49, Princeton Univ. Press, 1962.

[13] Walker, G., Estimates for the complex and quaternionic James numbers, Quart. J. Math. Oxford (2), 32 (1981), 467-489. 